\title{
Real Time System Based Monitoring of Turbine Parameters and Protection System in Thermal Power Plant
}

\author{
M. Surekha ${ }^{1}$, N. Suthanthira Vanitha ${ }^{2}$, K. Yadhari $^{3}$ \\ ${ }^{1}$ Student, Embedded System Technologies, Knowledge Institute of Technology, Salem, India \\ ${ }^{2}$ Head of the Department, Department of Electrical \& Electronics Engineering, Knowledge Institute of Technology, Salem, India \\ ${ }^{3}$ Assistant Professor, Department of Electrical \& Electronics Engineering, Knowledge Institute of Technology, Salem, India \\ Email address: \\ surekha.ece14@gmail.com (M. Surekha), hod.eee@kiot.ac.in (N. Suthanthira Vanitha), kyeee@kiot.ac.in (K. Yadhari)
}

\section{To cite this article:}

M. Surekha, N. Suthanthira Vanitha, K. Yadhari. Real Time System Based Monitoring of Turbine Parameters and Protection System in Thermal Power Plant. International Journal of Science, Technology and Society. Vol. 3, No. 3, 2015, pp. 69-75.

doi: $10.11648 /$ j.jijsts.20150303.11

\begin{abstract}
Currently in thermal power plant the turbine system parameters like temperature, speed, lubrication oil level and vibrations can be monitored by using the MATLAB. If any problem occurs in the turbine parameters, that can be controlled manually and the protection system is based on the relay mechanism which causes failure in the action of switch. In the proposed system, the drawbacks are eliminated with the support of high speed embedded processor. Real Time processor can be easily altered according to the ports and number of devices can also be connected. The parameters in the turbine system can be measured, monitored and controlled automatically. The parameters variations can be graphically represented by using the LabVIEW.
\end{abstract}

Keywords: ARM8Processor, MATLAB, LabVIEW, Automation System and Sensors

\section{Introduction}

Normally, the turbine system is a rotary mechanical device that extracts energy from fluid flow and it generates the power. If the turbine parameters move to an abnormal condition, it will cause a very high damage. In this paper, it mainly focuses on monitoring and controlling of the turbine system parameters. In the existing system, all the parameters in the turbine systemcan be monitored by using the MATLAB and it can be done by using the manual process. In order to overcome the drawbacks in the existing system the proposed system is employed.

The advanced ARM8 processor is used which is the heart of the system that controls all sub devices connected across it. The advanced processor is a flash type reprogrammable memory which has some peripheral devices to play this system as efficient. For the monitoring and controlling the parameters, temperature sensor, speed sensor, level sensor and vibration sensor is used to sense the temperature, speed, lubrication oil level and vibrations in the turbine system respectively. By using the embedded processor these four parameters can be monitored and it can be controlled automatically and graphically represented by using
LabVIEW.

\section{Literature Survey}

Krishna Prasad Dasari, Dr.A.M.Prasad[1] described the main aim of the research paper is to evaluate the method of environmental impact of power plant discharge by reducing the temperature difference between effluent and costal water and flow control. Water temperature control and flow control measurement have been designed in advance technology of industrial control area for thermal discharge model test. Digital temperature sensors, level sensors, Flow meters, different modulated circuits, dedicated interface are used in the test and controlling of the system is adopted in software designing and programming. Measurement procedure, data processing and controlling are done by Proportional Integral - Derivative (PID) controller. This technology can be implemented where the thermal effluent are discharged in coastal areas.

ShiyamSundar[2] described the paper deals with the Laboratory Virtual Instrumentation Engineering Workbench (LabVIEW) are widely used in industry for supervisory control and data acquisition of industrial processes. This paper described simulation of system for laboratory based 
thermal power plant generator setup using LabVIEW data logging and supervisory control (DSC) module. Using the input, output and functional parameters the simulation of generator unit and alarm handling technique is achieved.

Peng Guo and David infield[3] describedthe condition monitoring can greatly reduce the maintenance cost for a wind turbine. In this paper, a new condition- monitoring method based on the nonlinear state estimate technique for a wind turbine generator is proposed. The technique is used to construct the normal behavior model of the electrical generator temperature. Generator incipient failure is indicated when the residuals between model estimates and the measured generator temperature become significant. Moving window averaging is used to detect statistically significant changes of the residual mean value and standard deviation in an effective manner; when these parameters exceed predefined thresholds, an incipient failure is flagged. It is demonstrated that the technique can identify dangerous generator over temperature before damage has occurred that results in complete shutdown of the turbine.

Mohamed Zahran and Ali Yousef[4] described the purpose of the work is to investigate a monitoring of autonomous photovoltaic battery wind turbine hybrid system (PVBWHS). In this paper an intelligent graphical user interface (GUI) is built in sub-menus for system characterization. The PVBWHS modeling, simulation, and performance monitoring are also introduced. A LabVIEW model is designed whereby the hybrid system components are simulated as virtual instruments [VI] interacted with functional blocks. The developed monitoring system measures continuously the available power generated from the solar array and wind turbine, and the functional VI compare this with the actual load demand on real time estimates the storage battery operation mode.

Alberto Borghetti and MauroBosetti[5] described the paper presents a procedure for parameter identification along with its application to the model of a combined cycle power plant that includes the surrounding electrical network, built for the analysis of islanding manoeuvres transients. The paper illustrates both the power system computer model, implemented within the EMTP-RV simulation environment, and the developed identification procedure based on the interface between the developed model and MATLAB. The parameter identification procedure is applied to experimental transient recordings that make reference to a similar power plant.

\section{Existing System}

In the existing system, the turbine parameters like temperature, speed, lubrication oil level and vibrations in the thermal power plant can be monitored and controlled by using the MATLAB. It is a sophisticated data structures, contains built-in editing and debugging tools and supports object-oriented programming. All these parameter can be amplified and controlled the output that match with the input signal. If any problem occurs in the turbine parameters, it can be monitored and controlled by using the manual process. The protection system is based on the relay mechanism. The relays are connected with the pressure switches and it has lot of drawbacks such as occurrence of drift in the switch, failure in the action of the switch. There is no waveform representation and automation process was available in the existing system.

\section{Proposed System}

In order to overcome the problem in the existing system, the conventional method of the proposed system is employed The proposed system is to monitor and control the turbine parameters by using the ARM8 processor. The advanced processor operates at very low voltage and it consumes less power and it can be easily altered and number of devices can be connected according to the input and output ports. The embedded processor is used for real time monitoring of data. The parameters in the turbine system can be monitored and controlled by using the advanced processor.For the monitoring of temperature, speed, vibration and lubrication oil level, the temperature sensor, speed sensor, vibration sensor and level sensor can be used. All these parameter output is given to the amplifier unit. The output of the amplifier is then given to the advanced processor. PC is connected to the processor via RS 232 and it is a serial communication cable. So the parameters can be monitored and controlled by using the embedded processor. Whenever lubrication oil level becomes low, automatically it activates the relay to turn on the DC pump. Speed can be set as constant of 3000 RPM and according to the speed, the vibration can be controlled. Temperature is controlled with the help of cooling fan. By using the advanced ARM8 processor all these four parameters are monitored and it can be controlled automatically. The parameters variations can be graphically represented by using LabVIEW.

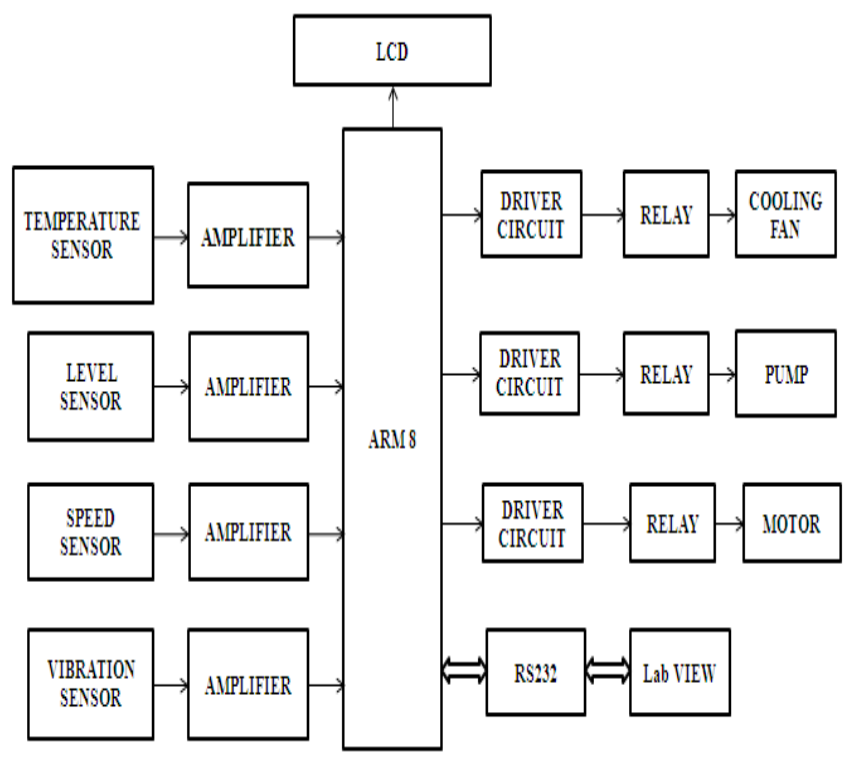

Fig. 1. Block Diagram of Proposed System. 
The system mainly operated by ARM8 processor. This paper consists of level sensor, temperature sensor, speed sensor and vibration sensor and they are used to measure the various parameters. The sensors can be connected to the amplifier which amplifies the signal.In the advanced processor, it converts the analog to digital signal through inbuilt ADC. It can be given to the driver circuit and it is used to convert the signal in the required form of processor.The signals can be connected to the relay and it is an electrically operated switch and current flowing through the coil of the relay creates a magnetic field which attracts a lever and changes the switch contacts.So by using the relay, the parameters can be controlled. If the temperature can be raised to higher level, it can be reduced by using the cooling fan. When the oil level is decreased to lower level, it can take oil automatically from the tank. When the speed increases, the turbine will off automatically. According to the speed, the vibration of turbine can be maintained. These parameters are implemented by using the advanced processor. It can be graphically represented by using the LabVIEW.

ARM8

ARM8 processor is used to monitor and control of the parameters automatically. According to the input and output ports, the devices can be connected to the processor. The advanced processor consists of the 64 pins. By using the pin diagram the sensors can be connected and control the parameters. The port1 is connected to the temperature, speed, vibrations and lubrication oil level sensor. The port0 is connected to the cooling fan, pump, and motor. According to the connections, the operations can be performed. Power supply is connected to the pin 10 and 11 . The parameters at normal and abnormal conditions can be displayed by using the LCD.

\section{LabVIEW}

All the parameter variations like temperature, speed, lubrication oil level and vibrations can be done by using the comparator. We have to set the limit value for these parameters. If the parameters can be raised above to that particular limit value, it can be represented as high in the front panel. Otherwise, it can be mentioned as normal.If the speed can be raised above 3000 RPM, it indicates the abnormal condition. It also indicates the speed variations by using the graphical method.

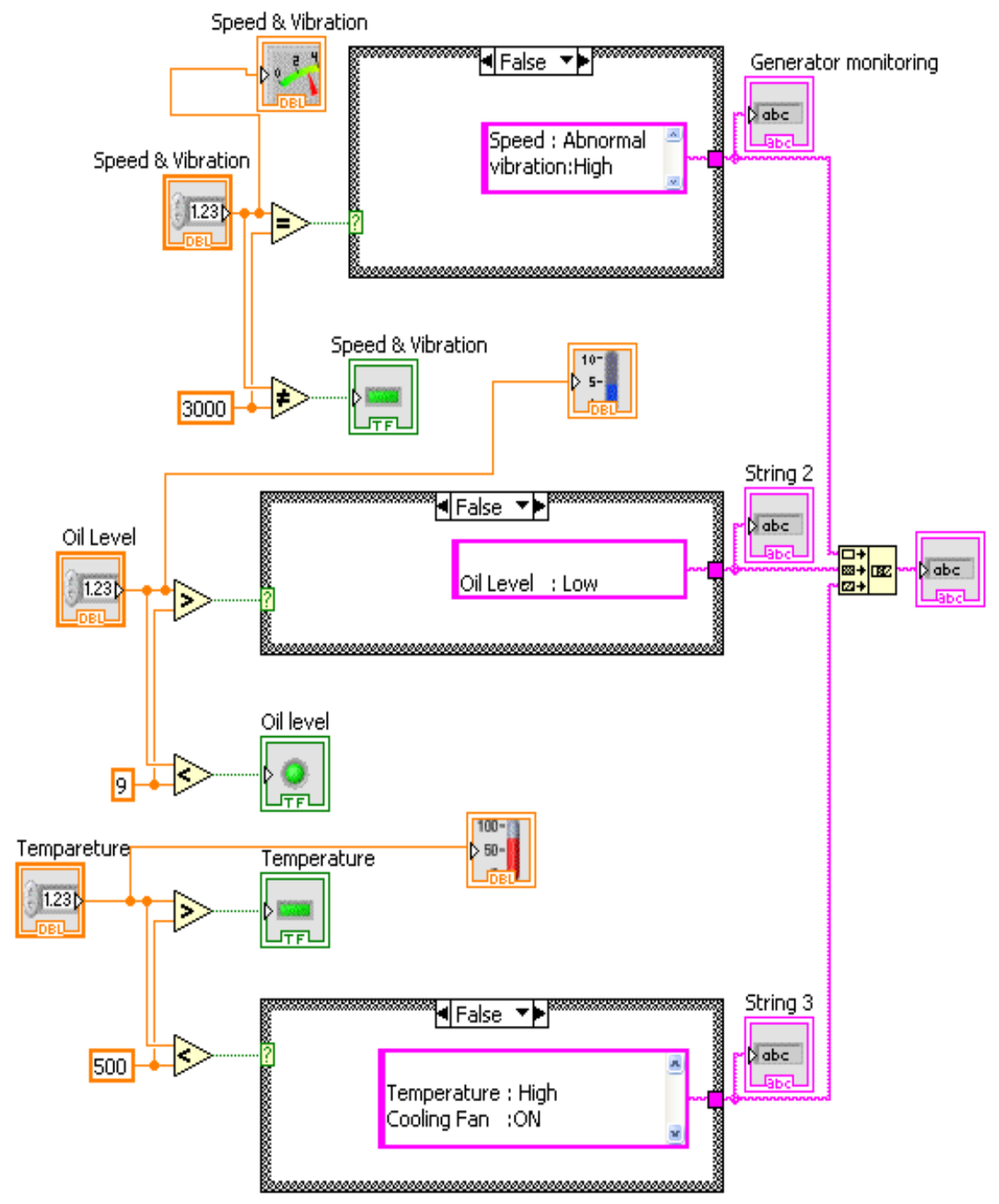

Fig. 2. Proposed Block Diagram of LabVIEW. 
If the speed is maintained as 3000 RPM, then it shows as normal condition. So according to the speed, the vibrations can be varied. If the speed can be raised to higher level, vibrations also can be raised. If the speed can be maintained as constant, vibrations will be normal in the turbine system. So we have to maintain the speed as constant. The lubrication oil level can be represented by using the tank as graphical method. If the lubrication oil level can be decreased to lower level, it can be mentioned as low and also the tank level will be represented as lower level. If the lubrication oil level can be maintained as constant, it can be mentioned as normal and the tank level will be represented as constant level in the front panel. The temperature can be represented by using the thermistor. If the temperature can be raised to higher level, it can be displayed as temperature is high. Then automatically the cooling fan will be ON. These variations can be represented in the graphical of thermistor. If the temperature cannot be raised to higher level, it can be displayed as temperature is normal. Then automatically the cooling fan will be OFF. All these conditions of the parameters can be graphically monitored and controlled by using LabVIEW.

\section{Flow Chart}

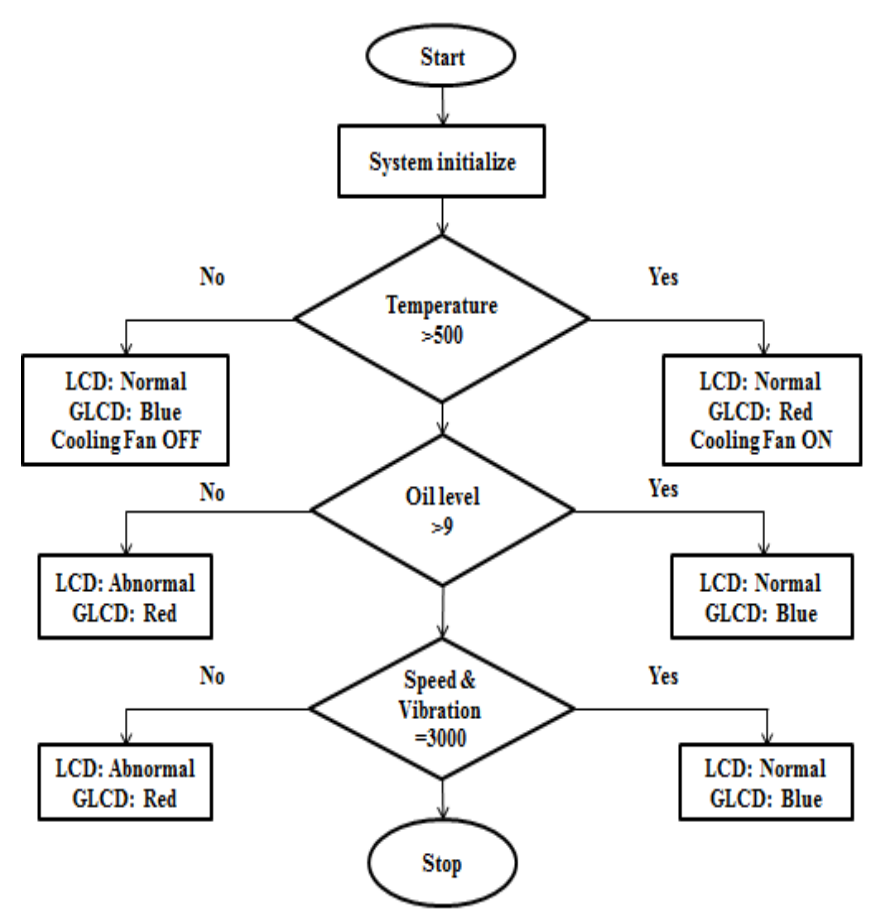

Fig. 3.Flow Chart of Proposed System.

\section{Algorithm}

Step 1: Start the process.

Step 2: Initialize the system.

Step 3: Representation of the temperature and according to the temperature it indicates the normal and abnormal conditions.

Step 4: The cooling fan will be automatically activated when the temperature moves to higher level.
Step 5: Representation of the speed, vibrations and lubrication oil level according to that the normal and abnormal conditions will be displayed.

Step 6: Stop the process.

\section{Results}

\subsection{Hardware Results}

It represents the monitoring and control of the parameters in the turbine system by using the ARM processor. In the ARM8 processor, the control panel consist of four options are temperature sensor, speed sensor, level sensor and vibration sensor. When selecting the option 1, it represents the temperature. If the temperature can be raised to higher level, automatically the cooling fan will be moved to ON condition. When selecting the option 2, it represents the speed. If the speed can be raised to higher level, it automatically represents as abnormal condition and it will reduce the speed.When selecting the option 3, it represents the level of lubrication oil.If the oil level decreases to lower level, it automatically represents as abnormal condition. When selecting the option 4, it represents the vibration level. If the vibration is in normal condition, it represents as normal.

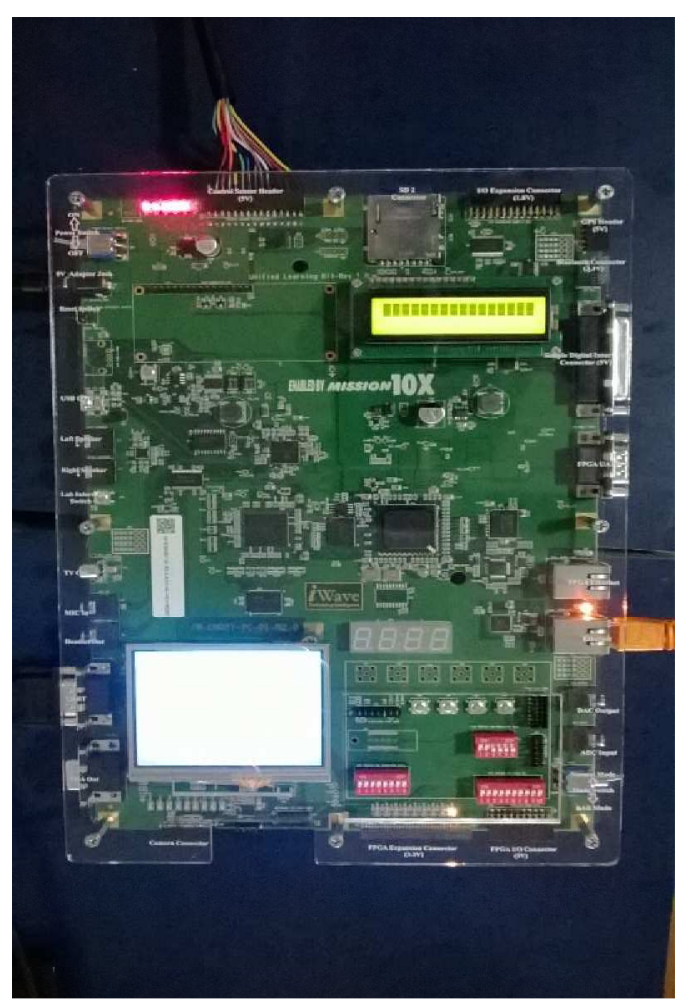

Fig. 4. ARM8 Processor Output.

\subsection{Software Results}

It indicates the parameters are in normal or abnormal condition by LED and graphical representation by using LabVIEW. In the fig.5, it represents the normal condition of 
the parameters. The speed can be maintained as 3000 , according to that vibration also maintained. It indicates the oil level as 10 and temperature as 400 . It can be graphically represented and displayed the parameters is in normal condition. In the fig. 6 , it represents the abnormal condition of parameters. The speed can be exceeding to 5000 and according to that the vibration can be varied. The oil level decreases to low and the temperature can be exceeding to 700 It can be represented by using the graphical method and displayed the parameters is in abnormal condition.

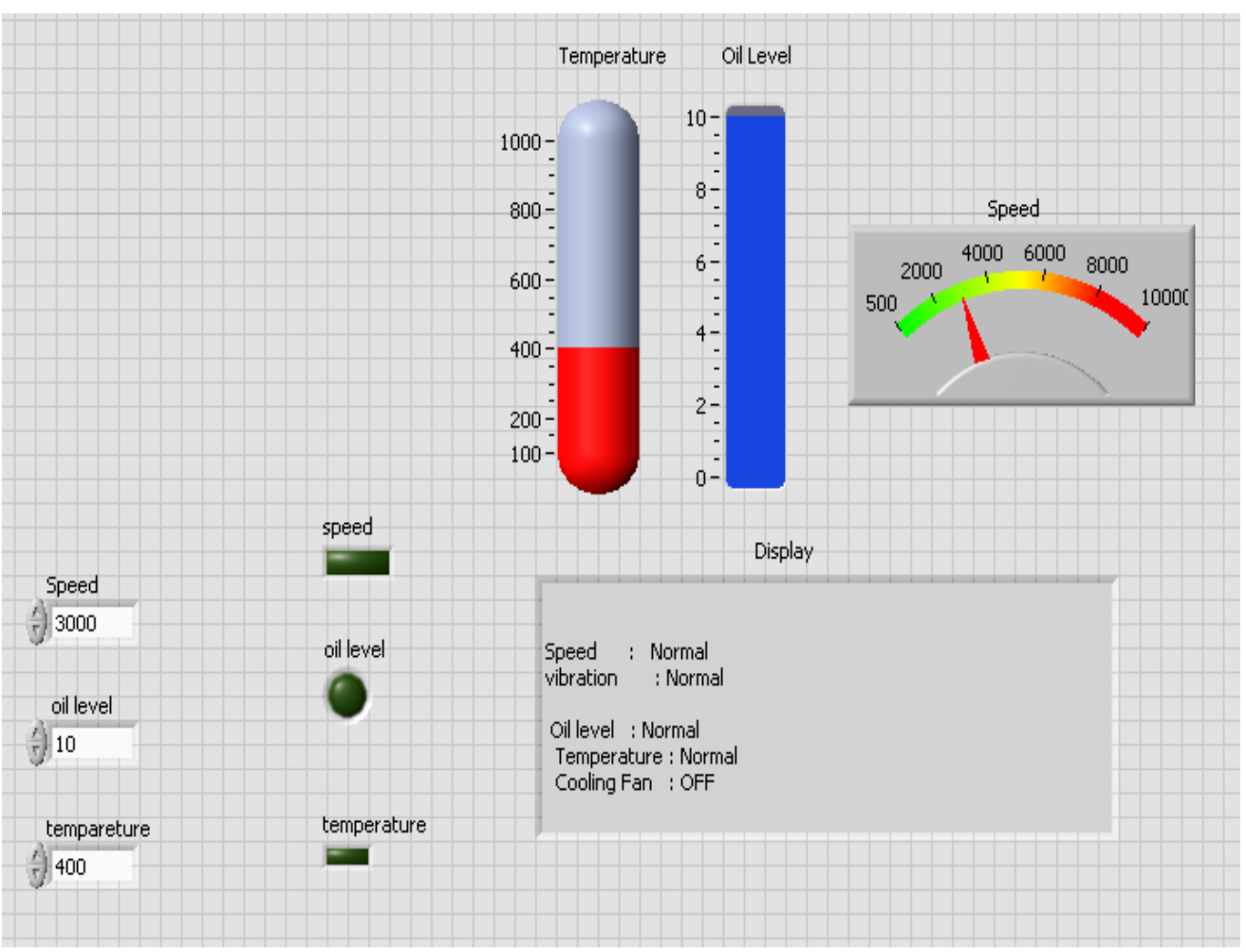

Fig. 5. Normal Condition of Parameters.

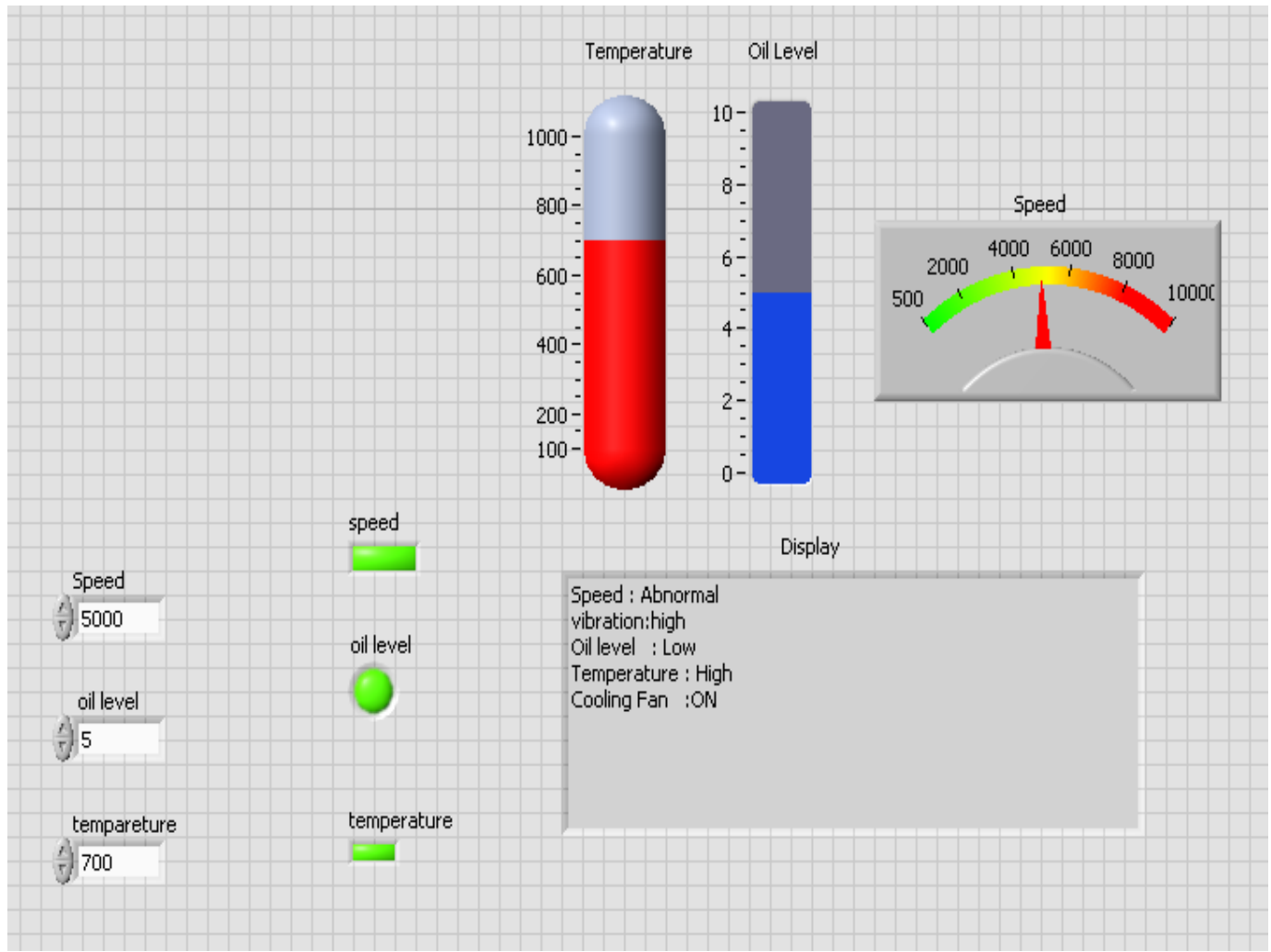

Fig. 6. Abnormal Condition of Parameters. 


\subsection{Results Comparison}

By comparing the hardware and software results, the parameters like temperature, speed, lubrication oil level and vibration can be monitored and controlled automatically. By using the ARM processor of eclipse software, the parameters at normal and abnormal condition can be represented by using the LCD and GLCD. GLCD (Graphical Liquid Crystal Display) is used to represent the variations in the color format.If the parameter is in normal condition, it indicates the blue color in GLCD and the message is displayed in LCD. If the parameter is in abnormal condition, it indicates the red color in GLCD and the message is displayed in LCD. In the LabVIEW, all these parameter variations can be represented by using the graphical method. If the parameters can be raised to higher or lower level, it can be displayed in the front panel. So by comparing both the eclipse software and LabVIEW, LabVIEW is easy to identify the variations of parameters by using the graphical method. By using the eclipse software, it is also very easy to identify the variation of parameters by using the color representation of GLCD.

\section{Conclusion}

In this project the turbine parameters like temperature, speed, lubrication oil level and vibrations can be monitored and controlled automatically by using ARM8 processor. The parameter variations of normal and abnormal conditions are represented by using the advanced processor and graphically represented by using LabVIEW. The man power is reduced and it reduces the damage that can be occurring in the turbine system. In future, the number of parameters will be added in the system for various applications. The parameters of the turbine system are temperature, speed, lubrication oil level and vibration can be monitored and controlled by using the online system. With the support of the wireless technology like GSM, it helps to prevent the damage occur in the turbine system.

\section{Coding}

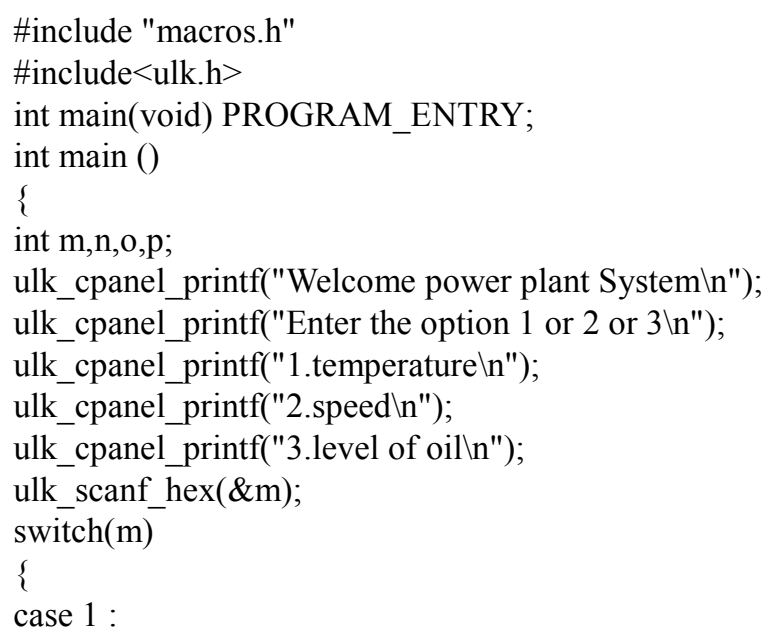

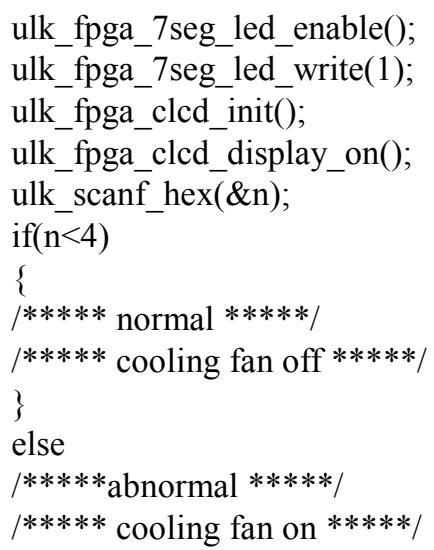

\section{References}

[1] Mohamed Zahran and Ali Yousef et al published a paper on "monitoring of photovoltaic wind turbine battery hybrid system", (WSEAS-2014). 
[2] ShiyamSundar published a paper on "simulation of generator unit in thermal power plant using LabVIEW", (IOSR-2013).

[3] PengGuo and David Infield published a paper on "wind turbine generator condition monitoring using temperature trend analysis", (IEEE-2012).

[4] Krishna Prasad Dasari and Dr.A.M.Prasad et al published a paper on "water temperature and flow control measurement for thermal discharge model using PID controller, (IJERA2012).

[5] Alberto Borghetti and Mauro Bosetti et al published a paper on "parameters identification of a power plant model for the simulation of islanding transients", (U.P.B.SCI.BULL-2010).

[6] Jaishree.S and Dr.K.Sathiyasekar et al published a paper on "wireless fault detection and preventive system for small wind turbine", (IJAREEIE-2014).

[7] L.SenthilMurugan and K.S.Aswath Rangaraj et al published a

\section{Biography}

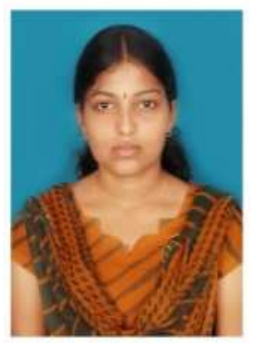

M. Surekha is pursuing, $P G$ in the discipline of Embedded System Technologies at Knowledge Institute of Technology, Salem, under Anna University, Chennai, India. She received her UG degree in the discipline of Electronics and Communication Engineering at Excel Engineering College, Komarapalayam under Anna University, Chennai, India. She has published and presented a number of technical papers in National Conferences and Technical symposiums. She is doing minor research works on various fields like PLC, Embedded Systems, and VLSI technology. She got best project award in ISTE for her project in UG. She is highly appreciated by the Head of the Department.

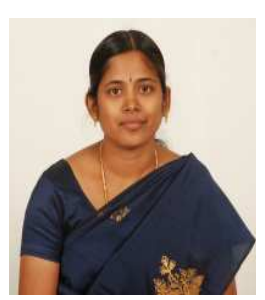

Dr. N. Suthanthira Vanitha is currently working as a Professor and Head of the Department of EEE at Knowledge Institute of Technology, Salem. She received the B.E. - Electrical and Electronics Engineering from K.S.R. college of Tech, Tiruchengode in 2000 from Madras University, M.E. Applied Electronics in Kamaraj University and Ph.D., in Biomedical Instrumentation \& Embedded Systems in 2009 from Anna University, Chennai. Her research interests lie in the area of Robotics, DSP, MEMS and Biomedical, Embedded Systems, Power Electronics and Renewable Energy systems, etc. She has published and presented number of technical papers in National and International Journals and Conferences. She has guided number of Projects for UG and PG students, currently guiding $12 \mathrm{Ph} . \mathrm{D}$., scholars. paper on "design of monitoring system and fault diagnosis in wind turbine based on can bus using arm", (IJIRSET-2014).

[8] Anish Mathew k et al published a paper on "internal model control of pressure process using arm microcontroller", (ICCEET-2012).

[9] Nie Chun-yan and Xu shan-shan et al published a paper on "data acquisition and realization of communication transmission based on LabVIEW", (IEEE-2012).

[10] V.Rukkumani and D.AngelineVijula et al published a paper on "Multiple parameter Monitor and Control in Wind Mills", (IJCAT-2012).

[11] John Sander et al published a paper on "steam turbine oil challenges", (LELUBRICANTS-2012).

[12] Masaki Kato and Seiichi Asano et al published a paper on "recent technology for reusing aged thermal power generating units", (FUJIELECTRIC-2010).

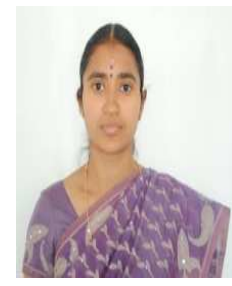

K. Yadhari is an Assistant Professor in the Department of Electrical and Electronics Engineering at Knowledge Institute of Technology, Salem. She had received B.E degree in Electrical and Electronics Engineering and M.E degree in Power System Engineering under Anna University, Chennai. She presented papers in National and International level conferences. She also published a journal at International level. She is the department Alumni Coordinator at Knowledge Institute of Technology, Salem. Her areas of interest are Unit commitment, Renewable energy systems and Distributed Generation. 